\title{
Gastric cancer surgery
}

\author{
Giovanni de Manzoni ${ }^{1} \cdot$ Han-Kwan Yang ${ }^{2}$
}

Received: 17 May 2018 / Accepted: 25 May 2018 / Published online: 13 June 2018

(C) Italian Society of Surgery (SIC) 2018

Gastric cancer is still one of the major causes of cancerrelated death worldwide [1]. In the last few years, many progresses have been made in the bio-molecular characterization [2,3] and management of this tumour [4] with the hope of improving the outcome of affected patients in the near future.

In this special issue of Updates in Surgery dedicated to gastric cancer, we aimed to provide a comprehensive synopsis of the recent advances and current issues in the diagnosis and treatment of this complex disease.

We selected authors among the global leaders in this field, each of whom was asked to address his own topic of major interest. Reviews and original articles deal with minimally invasive techniques, application of ERAS protocols, advances in multimodal therapy, newly proposed concepts of surgery in metastatic disease and possible clinical application of recent molecular classifications of gastric cancer.

As Guest Editors, we deeply thank the Editors of the journal for giving us this relevant chance and we hope that the selected articles will be of interest to the readers of Updates in Surgery.

\section{Compliance with ethical standards}

Conflict of interest There are no conflicts of interest to declare.

Research involving human participants and/or animals The research does not involve human participants and/or animals.

Informed consent There was no need to get informed consent.

\section{References}

1. Ferlay J, Soerjomataram I, Dikshit R, Eser S, Mathers C, Rebelo M (2015) Cancer incidence and mortality worldwide: sources, methods and major patterns in GLOBOCAN 2012. Int J Cancer 136(5):E359-E386

2. Cancer Genoma Atlas Research (2014) Comprehensive molecular characterization of gastric adenocarcinoma. Nature 513:202-209

3. Cristescu R, Lee J, Nebozhyn M et al (2015) Molecular analysis of gastric cancer identifies subtypes associated with distinct clinical outcomes. Nat Med 21:449-456

4. De Manzoni G, Marrelli D, Baiocchi GL, Morgagni P, Saragoni L, Degiuli M et al (2017) The Italian Research Group for Gastric Cancer (GIRCG) guidelines for gastric cancer staging and treatment: 2015. Gastric Cancer 20(1):20-30

The article is part of topical collection on Gastric Cancer Surgery.

Giovanni de Manzoni

giovanni.demanzoni@univr.it

1 General and Upper GI Surgery Division, University of Verona, Piazzale Aristide Stefani 1, 37126 Verona, Italy

2 Division of Gastrointestinal Surgery, Department of Surgery, Seoul National University Hospital, Seoul, Korea 